\title{
Multistage flow shop scheduling with bi-criteria objective using sequence dependent setup times
}

 \\ ${ }^{a}$ Apeejay College of Fine Arts, Jalandhar, Punjab, India \\ ${ }^{b}$ M.M. University, Mullana, Ambala, Haryana, India \\ ${ }^{c}$ D.A.V. College, Jalandhar, Punjab, India \\ Email: kknailwal@yahoo.co.in
}

\begin{abstract}
In many industrial setups, setup time on machines during the processing of given jobs in flow shop has great impact on the objective considered; hence the setups need to be taken separately during the process. Two types of setups exist in flow shop scheduling problem namely sequence-independent and sequence-dependent setup times. Scheduling of jobs with sequence-dependent setup times in flow shop scheduling is the most complicated case in the theory of scheduling. The main concern in this paper is the sequence-dependent setup time in which the setup time depends on the job type to be done and just completed. In this paper, a heuristic algorithm for solving bi-criteria flow shop problem with sequencedependent setup times is proposed. The bi-criteria objective considered is the minimization of the operational cost of machines subject to minimum makespan in a flow shop whenever the setup times on machines are sequence-dependent. As the problem is NP-hard, the proposed heuristic algorithm finds the latest time at which the machines should start so as to reduce their operating time and hence the operational cost subject to minimum makespan. The problem discussed in the paper can easily be seen in the industrial setups where expensive machinery is in operation or it is difficult to start or stop the machines due to operations technical limitations. The proposed algorithm makes use of famous NEH algorithm with a new tie-breaking rule. The tie-breaking rule breaks the tie for sequences with equal makespan during the run of algorithm. The starting of machines at latest time reduces the operating time and hence the operational cost of machines. The proposed heuristic algorithm when applied to given set of data reduced the total operational cost of machines from 2166 units to 2040 units subject to minimum makespan 90 units thereby reducing the total cost of machines by 126 units. A numerical example is also given to substantiate the algorithm.
\end{abstract}

Keywords: Scheduling, makespan, sequence-dependent setup time, operational cost, operational time 


\section{INTRODUCTION}

Scheduling is the allocation of resources over time to perform a collection of tasks to optimize one or more objectives. In general flow shop scheduling problem, $n$-jobs are to be scheduled on $m$-machines in some particular order and passing of jobs is not permitted (Baker 1974). The problem undertakes some assumptions: All jobs and machines are available at the beginning of processing. Pre-emption of jobs is not permitted. The machines are available throughout the processing and never breakdown. Each job is processed through each of the machine once and only once. The setup times are separated from processing time and are known. Each machine can perform only one task at a time. Machines may be idle.

The earliest research in the scheduling literature solves the two stage flow shop problem by giving the optimal solution for makespan minimization (Johnson 1954). The processing time of jobs included the setup times if any during the processing (Palmer 1965; Campbell el al. 1970; Nawaz et al. 1983). The NEH algorithm given by Nawaz et al. (1983) is considered to be the best heuristic algorithm for solving makespan as objective in flow shop scheduling. As mentioned they considered the setup times included in processing time of jobs. But in real situations of scheduling problems, there is a need for considering the separate setup times as it has great impact on the measure of performance considered. The setups times in scheduling theory can be grouped into two types; one being the sequence-independent setup times and second being the sequence-dependent setup times in which the setup times on machines depend upon the type of job to be processed and the type of job just processed. Allahverdi et al. (1999) surveyed the literature of scheduling problems involving setup times till 1999 and then the literature involving setup is again reviewed upto 2006 by Allahverdi et al. (2008). The other scheduling survey involving setup times include Yang (1999), Cheng et al (2000). Recently, Aqil and Allali (2018) presented three metaheuristics for minimization of makespan in flow shop involving sequence-dependent setup times. Sequence-dependent setup time is the most complicated case of setup times. The multi-purpose machines with different attributes mainly describe the environment of sequence-dependent setup times. Some industrial environments having sequence-dependent setups on machines are: In the production line of different types of gasoline the amount of setup time depends on the fuel to be manufactured and the fuel just produced; the cleaning and setting of the dyes for printing in printing industry is a case of sequence-dependent setup as processing of the job depends on its previously scheduled job. It may be due to any one of the reasons like difference in color ink, paper size and types used in the previous job, etc. Besides these the sequence-dependent setup times are found in stamping operation in plastic manufacturing and roll slitting in the paper industry (Yang 1999).

The concept of scheduling problems with sequence-dependent setup times has attracted many researchers and has become an emerging field due to its real life applications (Corwin and Esogbue 1974; Gupta and Darrow 1974; Rajendran and Ziegler 1997; Tan and Narasimhan 1997; Rios-Mercado and Bard 1999; Gagne et al. 2002; Rabadi et al. 2004; Gupta and Smith 2006; Mirabi 2011; Wang et al. 2017). These researchers considered problems in scheduling with single criteria involving sequence-dependent setup times. But in recent years, bi-criteria scheduling problem in flow shop with sequence dependent setup times has been an escalating attention of researchers and managers. Bi-criteria problems in scheduling are mainly categorized into two classes. In the class one, one out of the two criteria to be optimized is taken as objective subject to the second criteria as a constraint. In the second class, the two criteria to be optimized forms the objective function. Gupta et al. (2012, 2012a, 2013) formulated bi-criteria models belonging to first class for two stage flow shop with objective as minimization of makespan and rental cost with different parameters under the environment of sequence-dependent setup times. Mansouri et al (2009) considered two machine flow shop scheduling problem with sequence-dependent setup times using paretooptimality taking conflicting criteria as minimizing setups and makespan and it belongs to second group. Dhingra and Chandna (2010) developed a bi-criteria model for flow shop scheduling problem subject to the minimization of weighted sum of total weighted squared tardiness and makespan criterion. Eren (2010) also formulated bi-criteria $m$ machine scheduling in flow shop belonging to second group with aim of minimizing weighted sum of total completion time and makespan. Sharma et al. (2017) formulated a model falling in the first class of bi-criteria to optimize operational cost subject to minimum makespan. . The problem defined by Sharma et al. (2017) is a constrained flow shop problem for $n$-job and $m$-machine involving sequence-dependent setup times. Their bi-criteria model works only when the processing times of jobs on machines follow the condition $\min a_{i, s} \geq \max a_{i,(s+1)} ; s=1,2$, $3,4 \ldots \ldots \ldots m-2$. If this condition is violated the problem is out of scope of their model. In regards to this, we formulated the bi-criteria model belonging to class one with same objective functions with sequence-dependent setup times without constraint i.e. the proposed algorithm optimizes the operational cost of machines subject to minimum makespan in multi-stage flow shop with sequence-dependent setup times without any condition on 
Nailwal et al., Multistage flow shop scheduling with bi-criteria objective using sequence dependent setup times

processing times of jobs on machines. The rest of paper is organized as follows: Section 2 gives notations used throughout the paper; Section 3 presents the problem formulation; Section 4 describes the algorithm proposed to find the optimal sequence of jobs processing to solve the bi-criteria problem; Section 5 illustrates the steps of algorithm proposed with the help of numerical illustration and the conclusion is drawn in Section 6 followed by references.

\section{NOTATIONS}

The following notations have been used throughout the paper: $\sigma$ : Jobs sequence $1,2,3 \ldots n ; \sigma_{\gamma}$ : Sequence with minimum makespan; $J_{i}$ : $i$ th job, $i=1,2,3 \ldots n ; M_{l}: l$ th machine, $l=1,2, \ldots, m ; a_{i, l}$ : Time of processing of the $i$ th attribute on $M_{l} ; S_{i j, l}$ : Setup time if $i$ th job is in order just after $j$ th job on $M_{l} ; L_{l}\left(\sigma_{\gamma}\right)$ : Latest time when $M_{l}$ starts operating for $\sigma_{\gamma} ; C_{i j, l}\left(\sigma_{\gamma}\right)$ : Time to complete $i$ th job in order just after $j$ th job for $\sigma_{\gamma}$ on $M_{l} ; C_{i j, l}^{\prime}\left(\sigma_{\gamma}\right)$ : Time to complete $i$ th job in order just after $j$ th job for $\sigma_{\gamma}$ on $M_{l}$, when it starts processing at $L_{l}\left(\sigma_{\gamma}\right) ; O_{t_{l}}\left(\sigma_{\gamma}\right)$ : Operating time of $M_{l}$, when it starts processing at $L_{l}\left(\sigma_{\gamma}\right) ; O_{c_{l}}\left(\sigma_{\gamma}\right)$ : Operational cost of $M_{l}$ per unit time; $T_{c}\left(\sigma_{\gamma}\right)$ : Total operational cost for $\sigma_{\gamma}$ of the machines; $C_{n j, m}(\sigma)$ : Makespan

\section{PROBLEM FORMULATION}

With the assumptions on the flow shop environment, let the $i$ th job $(i=1,2 \ldots n)$ are processed through $m$-machines in the same order. The multipurpose $m$-machines possess different attributes for processing of jobs. Let $a_{i, l}$ be the processing time of $i$ th attribute on $M_{l} . S_{i j, l}$ be the required time to setup $M_{l}$ if $i$ th job is in order just after $j$ th job on $M_{l}$. The objective is to schedule $n$ - jobs in the sequence $\{\sigma\}$ so as to minimize the operational cost of the machines subject to minimum makespan. Table 1 to 3 represents the mathematical model of the problem in matrix form.

Table 1. Jobs with attributes on machines

\begin{tabular}{|c|c|c|c|c|c|c|c|}
\hline \multirow{2}{*}{} & \multirow{2}{*}{ Attributes } & \multicolumn{6}{|c|}{ Machine $\boldsymbol{M}_{\boldsymbol{l}}$} \\
\cline { 3 - 8 } & 1 & 1 & 2 & 3 & - & - & $f$ \\
\cline { 2 - 8 } & 1 & - & $J_{1}$ & - & - & - & - \\
\cline { 2 - 8 } & 2 & - & - & $J_{4}$ & - & - & - \\
\cline { 2 - 8 } & 3 & $J_{3}$ & - & - & - & - & - \\
\cline { 2 - 8 } & - & - & - & - & - & - & $J_{5}$ \\
\hline \multirow{2}{*}{} & - & - & - & - & $J_{2}$ & - & - \\
\hline & $\boldsymbol{e}$ & - & $J_{i}$ & - & - & - & $J_{n}$ \\
\hline
\end{tabular}

\begin{tabular}{|c|c|c|c|c|c|c|c|}
\hline & \multirow{2}{*}{ Attributes } & \multicolumn{6}{|c|}{ Machine $\boldsymbol{M}_{(++1)}$} \\
\cline { 2 - 8 } & 1 & 2 & 3 & - & - & $g$ \\
\cline { 2 - 9 } & 1 & $J_{3}$ & - & - & - & - & - \\
\cline { 2 - 9 } & 2 & - & $J_{1}$ & - & - & $J_{i}$ & - \\
\cline { 2 - 9 } & 3 & - & - & $J_{4}$ & - & - & - \\
\cline { 2 - 9 } & - & - & - & - & - & - & \\
\cline { 2 - 9 } & - & - & - & - & $J_{2}$ & - & - \\
\hline & $f$ & - & $J_{5}$ & - & - & - & $J_{n}$ \\
\hline
\end{tabular}

( $J_{i}$ is designated by its first attribute (row) on the one machine and second attribute (column) on the other machine)

Table 2. Processing time on machines

\begin{tabular}{|c|c|c|c|c|}
\hline Attributes & $\begin{array}{c}\text { Machine } \\
\boldsymbol{M}_{\boldsymbol{1}}\end{array}$ & $\begin{array}{c}\text { Machine } \\
\boldsymbol{M}_{\mathbf{2}}\end{array}$ & $\begin{array}{c}\text { Machine } \\
\boldsymbol{M}_{\boldsymbol{m}}\end{array}$ \\
\hline $\boldsymbol{1}$ & $a_{1,1}$ & $a_{1,2}$ & - & $a_{1, m}$ \\
\hline $\mathbf{2}$ & $a_{2,1}$ & $a_{2,2}$ & - & $a_{2, m}$ \\
\hline $\mathbf{3}$ & $a_{3,1}$ & $a_{3,2}$ & - & $a_{3, m}$ \\
\hline $\boldsymbol{-}$ & - & - & - & - \\
\hline $\boldsymbol{e}$ & $a_{e, 1}$ & $a_{e, 2}$ & - & $a_{e, m}$ \\
\hline $\boldsymbol{f}$ & - & - & - & - \\
\hline $\boldsymbol{f}$ & $a_{f, 1}$ & $a_{f, 2}$ & - & $a_{f, m}$ \\
\hline $\boldsymbol{g}$ & - & - & - & - \\
\hline & $a_{g, 1}$ & $a_{g, 2}$ & & $a_{g, m}$ \\
\hline
\end{tabular}

Table 3. Sequence-dependent setup time on $M_{l}$

\begin{tabular}{|c|c|c|c|c|c|c|c|c|}
\hline \multicolumn{2}{|c|}{} & \multicolumn{7}{|c|}{ Attributes } \\
\cline { 2 - 8 } \multicolumn{2}{|c|}{} & 1 & 2 & 3 & & $j$ & & $n$ \\
\hline \multirow{2}{*}{} & 1 & - & $S_{12, l}$ & $S_{13, l}$ & & $S_{1 j, l}$ & & $S_{I n, l}$ \\
\cline { 2 - 8 } & 2 & $S_{21, l}$ & - & $S_{23, l}$ & & $S_{2 j, l}$ & & $S_{2 n, l}$ \\
\cline { 2 - 9 } & 3 & $S_{31, l}$ & $S_{32, l}$ & - & & $S_{3 j, l}$ & & $S_{3 n, l}$ \\
\cline { 2 - 9 } & - & & & & - & & & \\
\cline { 2 - 9 } & $i$ & $S_{i l, l}$ & $S_{i 2, l}$ & $S_{i 3, l}$ & & $S_{i j, l}$ & & $S_{i n, l}$ \\
\cline { 2 - 8 } & - & & & & & & - & \\
\cline { 2 - 8 } & $n$ & $S_{n 1, l}$ & $S_{n 2, l}$ & & & $S_{n j, l}$ & & - \\
\hline
\end{tabular}


Nailwal et al., Multistage flow shop scheduling with bi-criteria objective using sequence dependent setup times

(If the attribute in row $i$ is in order just after the attribute in

Now, the problem mathematically can be defined as: Minimize

$T_{c}(\sigma)=\sum_{i=1}^{n} a_{i 1} \times O_{c_{1}}+O_{t_{2}}(\sigma) \times O_{c_{2}}+O_{t_{3}}(\sigma) \times O_{c_{3}}+----+O_{t_{m-1}}(\sigma) \times O_{c_{m-1}}+O_{t_{m}}(\sigma) \times O_{c_{m}}$

Subject to constraint: Minimum $C_{n j, m}(\sigma)$

\section{ALGORITHM PROPOSED}

The steps of proposed algorithm for solving bi-criteria multistage flow shop scheduling problem to optimize operational cost of machines subject to minimum makespan is given below. The proposed algorithm finds the latest times of machines at which they should start so as to minimize the operational cost of $m$-machines subject to minimum makespan. Before going to steps of algorithm, let us explain the tie-breaking rule used in the algorithm to break the tie in case the two or more jobs have the same makespan.

\subsection{Tie-breaking rule}

During the generation of sequences in the algorithm, more than one sequence may have the same makespan value. The tie is broken with the help of following procedure:

Calculate the operational cost of the machines for all the sequences with tie by the equation $T_{c}(\sigma)=\sum_{i=1}^{n} a_{i 1} \times O_{c_{1}}+O_{t_{2}}(\sigma) \times O_{c_{2}}+O_{t_{3}}(\sigma) \times O_{c_{3}}+----+O_{t_{m-1}}(\sigma) \times O_{c_{m-1}}+O_{t_{m}}(\sigma) \times O_{c_{m}}$. The sequence with the minimum operational cost is selected as the best sequence and is taken further for calculations. If a tie occurs again, sequence is randomly selected. This tie breaking rule will be applied during the run of the algorithm whenever we get more than one sequence with the same minimum makespan.

\subsection{Algorithm}

Step 1: Calculate $T_{i}$ for every job $J_{i}(\mathrm{i}=1,2,3 \ldots, n)$ on given m-machines given by the expression: $T_{i}=\sum_{j=1}^{m} a_{i, j}$

Step 2: Exhibit the job list according to the decreasing values of $T_{i}$ so obtained in step 1.

Step 3: Apply NEH algorithm (1983) to obtain the sequences $\sigma_{\gamma}$ having minimum makespan with regards to sequence-dependent setup times.

Step 4: Compute the latest time $L_{m}\left(\sigma_{\gamma}\right)$ of machine $m$ for sequence $\sigma_{\gamma}$ of jobs as

$L_{m}\left(\sigma_{\gamma}\right)=C_{n j, m}\left(\sigma_{\gamma}\right)-\sum_{i=1}^{n} a_{i, m}-\sum_{i=1}^{n-1} S_{i j, m}$

Step 5: For the sequence $\sigma_{\gamma}$ of jobs processing, evaluate: $Y_{1(m-1)}\left(\sigma_{\gamma}\right)=L_{m}\left(\sigma_{\gamma}\right)-a_{1,(m-1)}\left(\sigma_{\gamma}\right)$

$$
\begin{aligned}
& Y_{q(m-1)}\left(\sigma_{\gamma}\right)=L_{m}\left(\sigma_{\gamma}\right)-\sum_{i=1}^{q} a_{i,(m-1)}\left(\sigma_{\gamma}\right)-\sum_{i=1}^{q-1} S_{i j,(m-1)}\left(\sigma_{\gamma}\right)+\sum_{i=1}^{q-1} a_{i, m}+\sum_{i=1}^{q-2} S_{i j, m}\left(\sigma_{\gamma}\right) ; q=2,3, \ldots \ldots, n \\
& L_{m-1}\left(\sigma_{\gamma}\right)=\min _{1 \leq q \leq n}\left\{Y_{q(m-1)}\left(\sigma_{\gamma}\right)\right\}, O_{t_{m-1}}\left(\sigma_{\gamma}\right)=C_{n j,(m-1)}\left(\sigma_{\gamma}\right)-L_{m-1}\left(\sigma_{\gamma}\right)
\end{aligned}
$$

Now, for $l=(\mathrm{m}-1),(\mathrm{m}-2) \ldots \ldots . . .3$, enumerate $Y_{1(l-1)}\left(\sigma_{\gamma}\right)=L_{l}\left(\sigma_{\gamma}\right)-a_{1,(l-1)}\left(\sigma_{\gamma}\right)$,

$$
\begin{aligned}
& Y_{q(l-1)}\left(\sigma_{\gamma}\right)=L_{l}\left(\sigma_{\gamma}\right)-\sum_{i=1}^{q} a_{1,(l-1)}\left(\sigma_{\gamma}\right)-\sum_{i=1}^{q-1} S_{i j,(l-1)}\left(\sigma_{\gamma}\right)+\sum_{i=1}^{q-1} a_{i, l}\left(\sigma_{\gamma}\right)+\sum_{i=1}^{q-2} S_{i j, l}\left(\sigma_{\gamma}\right) ; q=2,3, \ldots \ldots ., n \\
& L_{3}\left(\sigma_{\gamma}\right)=\min _{1 \leq q \leq 6}\left\{Y_{q 3}\left(\sigma_{\gamma}\right)\right\}, O_{t_{l-1}}\left(\sigma_{\gamma}\right)=C_{n j,(l-1)}^{\prime}\left(\sigma_{\gamma}\right)-L_{(l-1)}\left(\sigma_{\gamma}\right)
\end{aligned}
$$

Step 6: Calculate total minimum operating cost of the machines for sequence $\left\{\sigma_{\gamma}\right\}$ of jobs

$$
T_{c}(\sigma)=\sum_{i=1}^{n} a_{i 1} \times O_{c_{1}}+O_{t_{2}}(\sigma) \times O_{c_{2}}+O_{t_{3}}(\sigma) \times O_{c_{3}}+----+O_{t_{m-1}}(\sigma) \times O_{c_{m-1}}+O_{t_{m}}(\sigma) \times O_{c_{m}}
$$

\section{NUMERICAL ILLUSTRATION}


Nailwal et al., Multistage flow shop scheduling with bi-criteria objective using sequence dependent setup times

To test the proposed algorithm the following illustration is carried out with the given data set. Consider five jobs to be processed on three machines. The attributes of jobs to be processed on three machines are given in the table 4 and 5. The processing times on the three machines for five jobs considered are given in table 6. Sequence-dependent setup times on $M_{1}, M_{2}$ and $M_{3}$ for five having different attributes are given in table 7 to 9 .

Table 4. Data set for attributes of jobs on machines $M_{1}$ and $M_{2}$

\begin{tabular}{|c|c|c|c|c|c|}
\hline \multirow{2}{*}{$\sum_{2}^{*}$} & \multirow{2}{*}{ Attributes } & \multicolumn{4}{|c|}{ Machine $\boldsymbol{M}_{\mathbf{2}}$} \\
\cline { 3 - 6 } & 1 & 1 & 2 & 3 & 4 \\
\cline { 2 - 6 } & 1 & - & $J_{1}$ & - & $J_{2}$ \\
\cline { 2 - 6 } & 2 & - & - & $J_{4}$ & - \\
\cline { 2 - 6 } & 3 & $J_{3}$ & - & - & $J_{5}$ \\
\hline
\end{tabular}

Table 5. Data set for attributes of jobs on machines $M_{2}$ and $M_{3}$

\begin{tabular}{|c|c|c|c|c|c|c|}
\hline \multirow{6}{*}{ 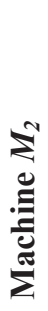 } & \multirow[b]{2}{*}{ Attributes } & & \multicolumn{4}{|c|}{ Machine $M_{3}$} \\
\hline & & 1 & 2 & 3 & 4 & 5 \\
\hline & 1 & - & $J_{3}$ & - & - & - \\
\hline & 2 & - & - & $J_{l}$ & - & - \\
\hline & 3 & - & - & - & $J_{4}$ & - \\
\hline & 4 & $J_{2}$ & - & - & - & $J_{5}$ \\
\hline
\end{tabular}

Table 6. Processing time of jobs on machines

\begin{tabular}{|c|c|c|c|}
\hline Jobs & Machine $\boldsymbol{M}_{\boldsymbol{1}}$ & Machine $\boldsymbol{M}_{\boldsymbol{2}}$ & Machine $\boldsymbol{M}_{\boldsymbol{3}}$ \\
\hline $\boldsymbol{J}_{\boldsymbol{1}}$ & 10 & 9 & 8 \\
\hline $\boldsymbol{J}_{\boldsymbol{2}}$ & 5 & 6 & 7 \\
\hline $\boldsymbol{J}_{\boldsymbol{3}}$ & 11 & 12 & 9 \\
\hline $\boldsymbol{J}_{\boldsymbol{4}}$ & 10 & 15 & 13 \\
\hline $\boldsymbol{J}_{\boldsymbol{5}}$ & 20 & 17 & 12 \\
\hline
\end{tabular}

Table 7. Data set for sequence -dependent setup

\begin{tabular}{|c|c|c|c|c|}
\hline & & \multicolumn{3}{|c|}{ Attributes } \\
\hline \multirow{4}{*}{ 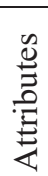 } & & $\mathbf{1}$ & 2 & 3 \\
\hline & 1 & - & 3 & 2 \\
\hline & 2 & 4 & - & 1 \\
\hline & 3 & 2 & 2 & - \\
\hline
\end{tabular}

(If attribute in row $i$ is in order just after attribute in column $j$ )

Table 8. Data set for sequence -dependent setup time on machine $M_{2}$

\begin{tabular}{|c|c|c|c|c|c|}
\hline & & & \multicolumn{3}{|c|}{ Attributes } \\
\hline \multirow{5}{*}{ 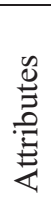 } & & 1 & 2 & 3 & 4 \\
\hline & 1 & - & 4 & 2 & 1 \\
\hline & 2 & 3 & - & 1 & 3 \\
\hline & 3 & 2 & 1 & - & 2 \\
\hline & 4 & 3 & 1 & 2 & - \\
\hline
\end{tabular}

(If attribute in row $i$ is in order just after attribute in column $j$ )

Table 9. Data set for sequence -dependent setup time on machine $M_{3}$

\begin{tabular}{|c|c|c|c|c|c|c|}
\hline & & & & \multicolumn{3}{|c|}{ Attributes } \\
\hline \multirow{6}{*}{ 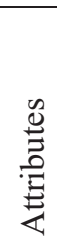 } & & 1 & 2 & 3 & 4 & 5 \\
\hline & 1 & - & 3 & 1 & 4 & 2 \\
\hline & 2 & 2 & - & 1 & 3 & 1 \\
\hline & 3 & 1 & 2 & - & 3 & 4 \\
\hline & 4 & 2 & 1 & 3 & - & 2 \\
\hline & 5 & 3 & 2 & 1 & 4 & - \\
\hline
\end{tabular}

(If attribute in row $i$ is in order just after attribute in column $j$ )

Let the operational cost per unit for the $M_{1}, M_{2}$ and $M_{3}$ be 9 units, 11 units and 13 units respectively. The task is to find the job sequence which gives minimum operational cost subject to minimum makespan.

Solution: As per step 1, the $T_{i}(i=1,2,3,4,5)$ values for jobs are 27,18,32,38 and 49. With step 2, we get the list with decreasing values of $T_{i}$ as $\{5,4,3,1,2\}$. Using NEH algorithm (1983) as per step 3, we get two sequences $J_{4}-J_{2}-J_{5}-$ $J_{3}-J_{1}$ and $J_{4}-J_{5}-J_{3}-J_{1}-J_{2}$ with minimum $C_{\max }=90$. The cost corresponding to these sequences are 2204 units and 2166 units

Table 10. Flow table of jobs for sequence $\sigma_{\gamma}$

\begin{tabular}{|c|c|c|c|}
\hline \multirow{3}{*}{ Jobs } & Machine $\boldsymbol{M}_{\boldsymbol{1}}$ & Machine $\boldsymbol{M}_{\mathbf{2}}$ & Machine $\boldsymbol{M}_{\boldsymbol{3}}$ \\
\cline { 2 - 4 } & $\begin{array}{c}\text { Flow In }- \\
\text { Out }\end{array}$ & $\begin{array}{c}\text { Flow In - } \\
\text { Out }\end{array}$ & $\begin{array}{c}\text { Flow In - } \\
\text { Out }\end{array}$ \\
\hline $\boldsymbol{J}_{\mathbf{4}}$ & $0-10$ & $10-25$ & $25-38$ \\
\hline $\boldsymbol{J}_{\boldsymbol{5}}$ & $12-32$ & $32-49$ & $49-61$ \\
\hline $\boldsymbol{J}_{\boldsymbol{3}}$ & $32-43$ & $50-62$ & $62-71$ \\
\hline $\boldsymbol{J}_{\boldsymbol{1}}$ & $45-55$ & $65-74$ & $74-82$ \\
\hline $\boldsymbol{J}_{\boldsymbol{2}}$ & $55-60$ & $75-81$ & $83-90$ \\
\hline
\end{tabular}

Table 11. Flow table of jobs for sequence $\sigma$

\begin{tabular}{|c|c|c|c|}
\hline \multirow{2}{*}{$J_{0 b s}$} & Machine $\boldsymbol{M}_{\boldsymbol{1}}$ & Machine $\boldsymbol{M}_{\mathbf{2}}$ & Machine $\boldsymbol{M}_{\boldsymbol{3}}$ \\
\cline { 2 - 4 } & $\begin{array}{c}\text { Flow In }- \\
\text { Out }\end{array}$ & $\begin{array}{c}\text { Flow In }- \\
\text { Out }\end{array}$ & $\begin{array}{c}\text { Flow In - } \\
\text { Out }\end{array}$ \\
\hline $\boldsymbol{J}_{\boldsymbol{4}}$ & $0-10$ & $12-27$ & $33-46$ \\
\hline $\boldsymbol{J}_{\boldsymbol{5}}$ & $12-32$ & $32-49$ & $50-62$ \\
\hline $\boldsymbol{J}_{\boldsymbol{3}}$ & $32-43$ & $50-62$ & $63-72$ \\
\hline $\boldsymbol{J}_{\boldsymbol{1}}$ & $45-55$ & $65-74$ & $74-82$ \\
\hline $\boldsymbol{J}_{\boldsymbol{2}}$ & $55-60$ & $75-81$ & $83-90$ \\
\hline
\end{tabular}


Nailwal et al., Multistage flow shop scheduling with bi-criteria objective using sequence dependent setup times

respectively. Applying the tie-breaking rule, the best sequence is the sequence with minimum operational cost say $\sigma_{\gamma}$ i.e. $\sigma_{\gamma}=J_{4}-J_{5}-J_{3}-J_{1}-J_{2}$ with regard to sequence-dependent setup time on machines. The In-Out flow table of jobs with due regards to sequence-dependent setup times for the sequence $\sigma_{\gamma}$ is given in table 10 .

As per step 4, $L_{3}\left(\sigma_{\gamma}\right)=t_{n j, 3}\left(\sigma_{\gamma}\right)-\sum_{i=1}^{n} a_{i, 3}-\sum_{i=1}^{n-1} S_{i j, 3}\left(\sigma_{\gamma}\right)=90-49-8=33$

Therefore, the operating time of machine $3, O_{t_{3}}\left(\sigma_{\gamma}\right)=C_{n j, 3}\left(\sigma_{\gamma}\right)-L_{3}\left(\sigma_{\gamma}\right)=90-25=57$ units

Enumerate the following values as per algorithm step 5, $Y_{12}=L_{3}\left(\sigma_{\gamma}\right)-a_{1,2}=33-15=18, Y_{22}=12, Y_{32}=15, Y_{42}=$ $13, Y_{52}=16$

Therefore, the latest time at which machine 2 is started $=L_{2}\left(\sigma_{\gamma}\right)=\min _{1 \leq q \leq 5}\left\{Y_{q 2}\left(\sigma_{\gamma}\right)\right\}=12$. Also, $C_{n j, 2}^{\prime}\left(\sigma_{\lambda}\right)=81$, $O_{t_{2}}\left(\sigma_{\lambda}\right)=C_{n j, 2}^{\prime}\left(\sigma_{\lambda}\right)-L_{2}\left(\sigma_{\lambda}\right)=81-12=69$

The flow table of jobs for the sequence $\sigma_{\gamma}$ with latest time is given in table 11. Here, the minimum total elapsed time, $C_{n j, 3}^{\prime}\left(\sigma_{\gamma}\right)=90$ units. Total minimum operational cost of machines= $T_{c}\left(\sigma_{\gamma}\right)=C_{n j, 1}\left(\sigma_{\gamma}\right) \times O_{c_{1}}+O_{t_{2}}\left(\sigma_{\gamma}\right) \times O_{c_{2}}+O_{t_{3}} \times O_{c_{3}}$ $=60 \times 9+69 \times 11+57 \times 13=2040$ units.

\section{CONCLUSION}

A bi-criteria multistage flow shop scheduling problem is considered with sequence-dependent setup time of the jobs for minimizing operational cost of machines subject to minimum makespan. The starting of machines for jobs processing if done at latest time $L_{m}\left(\sigma_{\gamma}\right)=C_{n j, m}\left(\sigma_{\gamma}\right)-\sum_{i=1}^{n} a_{i, m}\left(\sigma_{\gamma}\right)-\sum_{i=1}^{n-1} S_{i j, m}\left(\sigma_{\gamma}\right)$, it reduces the operating time and hence minimizes the total operational cost of machines. It is observed from the illustration given in support of the proposed algorithm that there is a effective decrease in the operational cost of machines subject to minimum makespan. The machine $M_{2}$ and $M_{3}$ when started at latest times $L_{2}=12$ and $L_{3}=33$ reduces the total cost of machines from 2166 units to 2040 units subject to minimum makespan of 90 units, thereby making the effective decrease by 126 units. Hence, the proposed algorithm is efficient in optimizing the bi-criteria involving operational cost and makespan. The study may further be extended by introducing some other conflicting objectives or can be extended to multi-criteria objectives.

\section{REFERENCES}

Allahverdi, A., Gupta, J. N., \& Aldowaisan, T. (1999). A review of scheduling research involving setup considerations. Omega, 27(2), 219-239.

Allahverdi, A., Ng, C. T., Cheng, T. E., \& Kovalyov, M. Y. (2008). A survey of scheduling problems with setup times or costs. European journal of operational research, 187(3), 985-1032.

Aqil, S., \& Allali, K. (2018, April). Three metaheuristics for solving the flow shop problem with permutation and sequence dependent setup time. In 2018 4th International Conference on Optimization and Applications (ICOA) (pp. 1-6). IEEE.

Baker, K.R. (1974). Introduction to Sequencing and Scheduling. Wiley: New York.

Campbell, H. G., Dudek, R. A., \& Smith, M. L. (1970). A heuristic algorithm for the $\mathrm{n}$ job, m machine sequencing problem. Management science, 16(10), B-630.

Cheng, T. E., Gupta, J. N., \& Wang, G. (2000). A review of flowshop scheduling research with setup times. Production and operations management, 9(3), 262-282.

Corwin, B. D., \& Esogbue, A. O. (1974). Two machine flow shop scheduling problems with sequence dependent setup times: A dynamic programming approach. Naval Research Logistics Quarterly, 21(3), 515-524.

Dhingra, A., \& Chandna, P. (2010). M-machine SDST flow shop scheduling using modified heuristic genetic algorithm. International Journal of Engineering, Science and Technology, 2(5), 216-225.

Eren, T. (2010). A bicriteria m-machine flowshop scheduling with sequence-dependent setup times. Applied Mathematical Modelling, 34(2), 284-293. 
Nailwal et al., Multistage flow shop scheduling with bi-criteria objective using sequence dependent setup times

Gagné, C., Price, W. L., \& Gravel, M. (2002). Comparing an ACO algorithm with other heuristics for the single machine scheduling problem with sequence-dependent setup times. Journal of the Operational Research Society, 53(8), 895-906.

Gupta, D., Sharma, S., Seema \& Nailwal, K.K. (2012). A bi-criteria two machine flowshop scheduling with sequence dependent setup time. International Journal of Mathematical Sciences. 11(3-4),183-196.

Gupta, D., Sharma, S., Seema and Nailwal, K.K. (2012). Bicriteria job block scheduling with sequence dependent setup time. Proceedings of International Conference on Optimization, Computing and Business Analytics. 8-13, India.

Gupta, D., Nailwal, K.K. and Sharma, S. (2013). Job block scheduling with dual criteria and sequence dependent setup time involving transportation times. Proceedings of International Conference on Computational Intelligence, Cyber Security and Computational Models. 321-329, India.

Gupta, J. N., \& Darrow, W. P. (1986). The two-machine sequence dependent flowshop scheduling problem. European Journal of Operational Research, 24(3), 439-446.

Gupta, S. R., \& Smith, J. S. (2006). Algorithms for single machine total tardiness scheduling with sequence dependent setups. European Journal of Operational Research, 175(2), 722-739.

Johnson, S. M. (1954). Optimal two-and three-stage production schedules with setup times included. Naval research logistics quarterly, $1(1), 61-68$.

Mansouri, S. A., Hendizadeh, S. H., \& Salmasi, N. (2009). Bicriteria scheduling of a two-machine flowshop with sequence-dependent setup times. The International Journal of Advanced Manufacturing Technology, 40(11-12), 1216-1226.

Mirabi, M. (2011). Ant colony optimization technique for the sequence-dependent flowshop scheduling problem. The International Journal of Advanced Manufacturing Technology, 55(1-4), 317-326.

Nawaz, M., Enscore Jr, E. E., \& Ham, I. (1983). A heuristic algorithm for the m-machine, n-job flow-shop sequencing problem. Omega, 11(1), 91-95.

Palmer, D. S. (1965). Sequencing jobs through a multi-stage process in the minimum total time-a quick method of obtaining a near optimum. Journal of the Operational Research Society, 16(1), 101-107.

Rabadi, G., Mollaghasemi, M., \& Anagnostopoulos, G. C. (2004). A branch-and-bound algorithm for the early/tardy machine scheduling problem with a common due-date and sequence-dependent setup time. Computers \& Operations Research, 31(10), 1727-1751.

Rajendran, C., \& Ziegler, H. (1997). A heuristic for scheduling to minimize the sum of weighted flowtime of jobs in a flowshop with sequence-dependent setup times of jobs. Computers \& Industrial Engineering, 33(1-2), 281-284.

Rios-Mercado, R. Z., \& Bard, J. F. (1999). A branch-and-bound algorithm for permutation flow shops with sequence-dependent setup times. IIE transactions, 31(8), 721-731.

Sharma, S., Gupta, D., \& Nailwal, K. K. (2017). Bi-criteria multistage flow shop scheduling with sequencedependent setup times. International Journal of Operational Research, 29(1), 127-147.

Tan, K. C., \& Narasimhan, R. (1997). Minimizing tardiness on a single processor with sequence-dependent setup times: a simulated annealing approach. Omega, 25(6), 619-634.

Yang, W. H. (1999). Survey of scheduling research involving setup times. International Journal of Systems Science, 30(2), 143-155.

Wang, Y., Li, X., \& Ma, Z. (2017). A Hybrid Local Search Algorithm for the Sequence Dependent Setup Times Flowshop Scheduling Problem with Makespan Criterion. Sustainability, 9(12), 2318. 